\title{
Problems and Countermeasures of Human Resources Training in Public Institutions
}

\author{
Li Haiping \\ Changqing Yellow River Bureau, Jinan Yellow River Bureau, Jinan, China
}

\section{Email address:}

35195341@qq.com

\section{To cite this article:}

Li Haiping. Problems and Countermeasures of Human Resources Training in Public Institutions. Science Innovation. Vol. 9, No. 4, 2021, pp. 164-166. doi: 10.11648/j.si.20210904.18

Received: May 20, 2021; Accepted: June 25, 2021; Published: July 9, 2021

\begin{abstract}
This paper takes the human resource training of public institutions as the object of analysis, firstly outlines the content and significance of human resource training of public institutions, and then analyzes the problems existing in human resource training of public institutions, finally, the paper discusses the countermeasures of Human Resource Training in institutions, in order to improve the quality of human resource training in institutions and improve the effectiveness of human resource training in institutions. The leaders of public institutions need to take the lead in renewing the concept, establish the strategic thinking of training, bring training into the planning of public institutions, improve the whole set of training system, actively develop training resources, and provide sufficient guarantee for training work, to cultivate a high-quality team of training teachers, so as to improve the quality of training, improve the effectiveness of human resources training institutions. Human Resources training can enhance the work skills of the staff of the administrative institutions, enrich the knowledge reserve of the staff of the administrative institutions, broaden the staff's horizons, and training can improve the staff's work efficiency, at the same time, it will also improve the work quality of administrative institutions. In order to enhance the staff's ability to perform their duties, the overall efficiency of the administrative and public institutions.
\end{abstract}

Keywords: Public Institutions, Human Resources, Training

\section{事业单位人力资源培训存在的问题与对策}

\section{李海平}

长清黄河河务局, 济南黄河河务局, 济南, 中国

\section{邮箱}

35195341@qq.com

摘要: 本文以事业单位人力资源培训为分析对象, 首先概述了事业单位人力资源培训内容与意义, 接着分析了事业单 位人力资源培训存在的问题, 最后论述了事业单位人力资源培训对策, 以便提升事业单位人力资源培训质量, 提高事 业单位人力资源培训实效。事业单位领导需要带头更新理念, 树立培训战略思想, 将培训纳入到事业单位规划之中, 完善整套培训体系, 积极开发培训资源, 为培训工作提供充足的保障, 培育一支高素质的培训师资队伍, 以此提升培 训的质量、提升事业单位人力资源培训的实效。人力资源培训可提升行政事业单位工作人员的工作技能, 丰富行政事 业单位工作人员的知识储备, 拓展工作人员视野, 培训可提高工作人员的工作效率, 同时也提高行政事业单位工作质 量。以此提升工作人员的履职能力、行政事业单位整体工作效率。

关键词：事业单位，人力资源，培训 


\section{1. 引言}

在事业单位人力资源管理中, 人力资源培训属于人力 资源管理的一项重要内容, 通过培训可提升事业单位人员 的工作素质, 以此提高事业单位人员工作质量, 完成各种 各样的工作 $[1,2]$ 。目前, 事业单位并没有树立起正确的人 力资源培训理念, 一些事业单位领导认为人力资源培训并 不重要, 没有将人力资源培训提升到事业单位发展规划之 中, 影响人力资源培训实效, 为此, 事业单位领导需要带 头更新理念, 树立培训战略思想, 将培训纳入到事业单位 规划之中, 以此提升事业单位人力资源培训的实效[3]。人 力资源培训可提升行政事业单位工作人员的工作技能, 丰 富行政事业单位工作人员的知识储备，拓展工作人员视 野, 以此提升工作人员的履职能力。因此, 事业单位需要 加强人员培训, 通过人员培训提升自身服务水平。

\section{2. 事业单位人力资源培训的概述}

行政事业单位人力资源培训主要是指行政事业单位 人力资源部门有计划、有目的的培训事业单位的工作人 员，使行政事业单位工作人员获得全新技能，以提升他们 履行职责的能力, 同时也提高行政事业单位的工作效率。 一般而言, 行政事业单位人力资源培训具有以下几个方面 的重要意义[4]。

第一，提升工作人员的履职能力。人力资源培训可提 升行政事业单位工作人员的工作技能, 丰富行政事业单位 工作人员的知识储备, 拓展工作人员视野, 以此提升工作 人员的履职能力。

第二，提高事业单位的工作效率。培训可提高工作人 员的工作效率，以此提高行政事业单位整体工作效率，同 时也提高行政事业单位工作质量。

\section{3. 事业单位人力资源培训存在的问题}

\section{1. 培训理念落后}

目前，事业单位并没有树立起正确的人力资源培训理 念, 一些事业单位领导认为人力资源培训并不重要, 因此 并没有将人力资源培训提升到事业单位发展规划之中, 影 响人力资源培训实效 $[5,6]$ 。与此同时, 许多员工也没有端 正自身态度, 并没有认识到培训对于自己的重要意义, 认 为培训只不过是走过场, 培训并不能够提高自身工作技 能，也无法提高自身工作效率，因此导致很多员工的培训 思维陈旧落后, 无法适应新形势下事业单位人力资源培训 要求[7]。

\section{2. 培训体系不完善}

第一, 没有做好培训需求分析。在开始培训之前, 培 训需求分析可以了解培训人员培训需求, 以提高培训的针 对性和有效性。目前, 事业单位在培训之前并没有做好培 训需求分析, 培训需求分析也只是简单的分析, 并没有深 入与一线工作人员进行交流, 致使培训需求分析是一种经
验性质的分析, 并不能够真实反映一线工作人员的真实培 训需求 $[8]$ 。

第二，缺乏培训监控。在培训的过程之中，事业单位 培训还需要加强培训监控, 通过培训监控可以端正培训人 员的培训态度, 可以提升培训人员的培训积极性。目前, 事业单位的培训缺乏培训, 监控培训并没有专业的监控老 师, 致使一些培训人员培训态度不端正, 认为培训只是走 过场的模式, 甚至有很多培训人员中途请假, 致使培训工 作流于形式，无法达到培训的目标。

第三，培训评估效果不理想。在培训的过程之中，评 估可以有效的分析培训的质量, 可以发现培训中出现的问 题, 以此提升培训的质量。目前, 事业单位的培训评估方 式并不科学, 评估的模式非常简单, 难以客观反映事业单 位培训的实效。在培训评估的过程之中, 评估的主体非常 单一, 只是由人力资源部门进行评估, 并没有邀请部门领 导进行评估, 至此培训评估的效果不是很理想, 难以真实 反映参训人员的培训质量[9]。

\section{3. 培训资源开发滞后}

第一，缺乏培训资金保障。在培训的过程之中，资金 是确保培训工作开展的重要基石, 充足的资金可以确保培 训工作有效开展。目前, 事业单位培训缺乏充足资金, 由 于缺乏充足的资金导致事业单位的培训工作时断时续, 最 终会影响事业单位培训工作的质量[10]。

第二, 培训课程滞后。在培训的过程之中, 培训课程 可以指导培训工作。目前, 事业单位的培训课程千篇一律, 培训课程并没有根据不同的岗位开设不同的内容, 难以满 足不同岗位员工的需求, 导致培训内容千篇一律, 影响培 训的质量。

\section{4. 缺乏高素质培训教师}

在人力资源培训的过程之中, 高素质的教师常常可以 提升培训的质量, 高素质的教师可以提升培训效果, 因此 一直合格的培训队伍对事业单位培训至关重要。目前, 事 业单位内部缺乏一支高素质的培训师资队伍, 现有的培训 师资力量是单位内部的中层干部, 而管理人员由于业务繁 忙并没有足够精力关注培训。事业单位的中层干部虽然了 解单位内部的业务, 但是他们并没有丰富的教学技巧, 因 此难以将自身的业务能力传递给其他人员, 影响事业单位 培训的质量。

\section{4. 事业单位人力资源培训的对策}

\section{1. 更新理念}

为提升事业单位人力资源培训的质量, 事业单位需要 更新培训理念, 以此提升事业单位培训的实效。为此, 事 业单位领导需要带头更新理念, 树立培训战略思想, 将培 训纳入到事业单位规划之中, 以此提升事业单位人力资源 培训的实效。与此同时, 事业单位的员工也需要端正自身 的态度, 更新自身的培训理念, 认识到培训对于自身发展 
的意义, 积极参与培训, 融入到培训氛围之中, 以此提升 培训的实效[11]。

\section{2. 完善培训体系}

第一, 加强培训需求分析。在培训之前, 事业单位人 力资源部需要与培训人员进行沟通, 了解培训人员真实的 培训需求, 以此制定培训大纲, 提高培训的针对性和有效 性, 提升培训的质量。

第二, 加强培训监控。在培训的过程之中, 人力资源 部门还需要做好培训的监控工作, 可以通过监控摄像头了 解培训现场的秩序, 同时还可以通过培训人员签到的模式 了解培训人员的出勤状况, 以促使培训人员更新自身的培 训理念, 端正自身的培训态度, 认识到培训的意义, 使培 训工作可以有效开展。

第三, 做好培训评估。为提升培训的质量事业单位, 在培训之后还需要做好培训评估工作, 通过培训评估工作 了解参与培训人员的培训实效, 以此提升培训的质量。在 培训评估的过程之中, 事业单位可以采用多元化的培训评 估方式, 同时也可以丰富培训评估主体以此提升培训评估 的实效。例如, 事业单位可以邀请培训人员的上级领导参 与到培训评估之中, 观察培训人员在培训之后的各项工作 表现, 以此分析培训的质量[12]。

\section{3. 积极开发培训资源}

为提升事业单位人力资源培训质量, 事业单位还需要 积极开发培训资源, 为培训工作提供充足的保障。

第一，筹集培训资金。为提升培训质量，事业单位需 要筹集培训资金, 事业单位可以在财政中列出专项培训资 金，同时还可以设置培训资金基金，以此为培训提供强有 力的资金支持，确保培训工作可以顺利开展。

第二, 积极开发培训课程。为提升培训实效, 事业单 位可积极开发各种培训课程, 可以针对不同的岗位设置不 同的课程内容, 以此提升培训课程的针对性, 确保不同岗 位的员工可以获得充足的培训, 提高培训的质量[13]。

\section{4. 打造高素质的培训团队}

为提升事业单位培训质量, 事业单位可培育一支高素 质的培训师资队伍, 以此提升培训的质量。为此, 事业单 位可加强培训团队的选拔工作, 同时还可以在社会上广泛 招聘培训师, 可以聘请一些专业人士担任全职培训师以此 打造之高素质的培训团队, 提升培训的质量。与此同时, 事业单位还可以将培训团队分成多个不同的层级, 不同层 级的培训团队享受不同的待遇, 以此激发培训团队培训的 积极性, 使培训师可以努力提高自身的培训技能[14]。

\section{5. 结语}

综上所述, 为加强事业单位人力资源培训, 事业单位 领导需要根据培训理念完善培训体系, 带头更新理念, 树 立培训战略思想, 将培训纳入到事业单位规划之中, 以此 提升事业单位人力资源培训的实效 [15]。事业单位人力资 源部需要与培训人员进行沟通, 了解培训人员真实的培训
需求, 以此制定培训大纲, 提高培训的针对性和有效性, 提升培训的质量。积极开发培训资源, 为提升培训质量, 事业单位需要筹集培训资金, 为培训提供强有力的资金支 持, 确保培训工作可以顺利开展。通过培训评估工作了解 参与培训人员的培训实效, 以此提升培训的质量。开发各 种培训课程, 可以针对不同的岗位设置不同的课程内容, 开发各种培训课程, 可以针对不同的岗位设置不同的课程 内容, 同时也可以培育一支高素质的培训团队, 以此提高 事业单位人力资源培训的质量, 加强事业单位人力资源培 训的实效。

\section{参考文献}

[1] 王卫东. 关于事业单位人力资源绩效考核[J]. 经营管理者, 2014.

[2] 孙秀君. 事业单位人力资源改进策略[J]. 现代营销学苑版, 2015.

[3] 郭影. 事业单位人力资源培训以及开发[J]. 大众投资指南, 2019.

[4] 宋水玉. 论提升行政事业单位内部控制有效性的策略 [J]. 中国总会计师, 2015.

[5] 胥译心, 冯阳. 当前事业单位人力资源培训问题与应对措 施[J]. 人力资源, 2020, No.459(06):76-77.

[6] 侯玉珍. 人力资源管理创新措施的探析 [J]. 中外企业家, 2016.

[7] 崔荣. 新形势下事业单位人力资源管理改革初探[J]. 人才 资源开发, 2014.

[8] 李叶萍. 事业单位人力资源培训与开发中的常见问题及对 策[]]. 人力资源, 2019, 000(009):179.

[9] 李烨. 事业单位人力资源培训面临的现状与应对措施 [J]. 商情, 2019, 000(024):279.

[10] 张吉峰. 论企业人力资源培训的重要性[J]. 中国高新技术 企业, 2011.

[11] 赵玄枝. 我国事业单位人力资源培训浅析 [J]. 现代经济信 息, 2014.

[12] 王浩业. 事业单位人力资源培训面临的挑战与应对措施[J]. 中国战略新兴产业, 2019, 000(018):227.

[13] 郑大鹏. 实施“职级管理”, 为校长专业发展铺设成功之路 [J]. 小学时代教师, 2012.

[14] 张力军. 加快率先实现农业现代化步伐 [J]. 吉林日报, 2016.

[15] 李强. 关于对事业单位人力资源绩效考核的探讨[J]. 中国 外资, 2014. 